Fig. 1.

\section{Ein Fall von Myxo-Fibrom des Felsenbeins mit multipler Hirnnervenlähmung. ${ }^{1}$ )}

Von Dr. D. Schwabach und Dr. M. Bielschowsky in Berlin. I.

M. H.! Der Fall, über dessen Krankheitsverlauf und Obduktionsbefund Herr Kollege Bielschowsky und ich Ihnen kurz berichten möchten, betrifft eine 50 jährige Frau, die wir im städtischen Krankenhause in der Gitschinerstraße hier zu beobachten Gelegenheit hatten. Sie klagte bei ihrer Aufnahme über reißende Schmerzen in der linken Seite des Kopfes und besonders im linken Ohr. Die Schmerzen sollen schon seit vier Wochen bestehen; wenige Tage nach deren Auftreten bemerkte Patientin, daß ihr Mund schief sei. Seit 14 Tagen leidet sie an Schwindelanfällen mit häufig sich wiederholender Uebelkeit und Erbrechen. Seit derselben Zeit merkt sie, daß sie auf dem linken Ohr schlecht hört; über subjektive Geräusche hat sie nicht zu klagen. Im weiteren Verlauf der Erkrankung gesellten sich zu den genannten Beschwerden Symptome, die auf eine Erkrankung der verschiedensten Hirnnerven hindeuteten, welche schließlich vom fünften bis inkl. zwölften befallen wurden. Ueber die Einzelheiten wird Herr Kollege Bielschowsky berichten. Beim Versuch zu gehen tritt leichtes Schwanken ein, das jedoch sofort aufhört, sobald durch Schließen eines Auges die infolge der Abducenslähmung bestehenden Doppelbilder ausgeschaltet werden. Spontaner Nystagmus besteht nicht. Die Untersuchung der Ohren ergab rechts nichts Abnormes; Hörfähigkeit nicht herabgesetzt, Trommelfellbefund normal. Links wird das Ticken der Uhr weder durch Luft- noch durch Knochenleitung perzipiert, weder Flüster- noch laute Sprache gehört. Die Prüfung mit Stimmgabeln ergibt vollständige Taubheit für alle Töne vom Contra $\mathrm{C}$ bis fisIV. Der Ton der auf den Scheitel aufgesetzten Stimmgabel (c) wird nach der gesunden (rechten) Seite lokalisiert; am linken Warzenfortsatz kommt derselbe Ton noch einen Augenblick zur Perzeption. Am Trommelfell eine ganz leichte Rötung, die aber nach einigen Tagen vollständig verschwunden war.

Daß hier eine Erkrankung des schallempfindenden Apparats vorlag, war nicht zu bezweifeln, und mit Rücksicht auf das Zusammenfallen der Hörstörungen mit der Affektion der verschiedensten Hirnnerven mußte man daran denken, daß es sich um eine gemeinsame intrakraniell gelegene Ursache handelte. Als solche glaubte man einen Tumor des Kleinhirnbrückenwinkels annehmen zu sollen. Bei der Obduktion der fast ein Jahr lang im Krankenhause verpflegten Patientin fand sich nun in der Tat eine basale Neubildung, aber nicht eine solche des Kleinhirnbrückenwinkels (Acusticustumor), sondern eine Neubildung, welche das ganze linke Felsenbein, das ich Ihnen hier vorlege und von dem ich Ihnen auch eine Photographie herumgebe (Fig. 1), einnahm: Es erscheint zunächst in seinem ganzen Umfange gegen die Norm vergrößert; außerdem sieht man an seiner vorderen Fläche zwei durch eine breite Furche voneinander getrennte halbkugelige, mit breiter Basis aufsitzende Tumoren, deren größerer (a) lateralwärts, nahe der Pars squamosa des Schläfenbeins, deren kleinerer (b) medialwärts gegen die Spitze des Felsenbeins hin

1) Nach einer Demonstration in der Berliner otologischen Gesellschaft am 4. Dezember 1908.

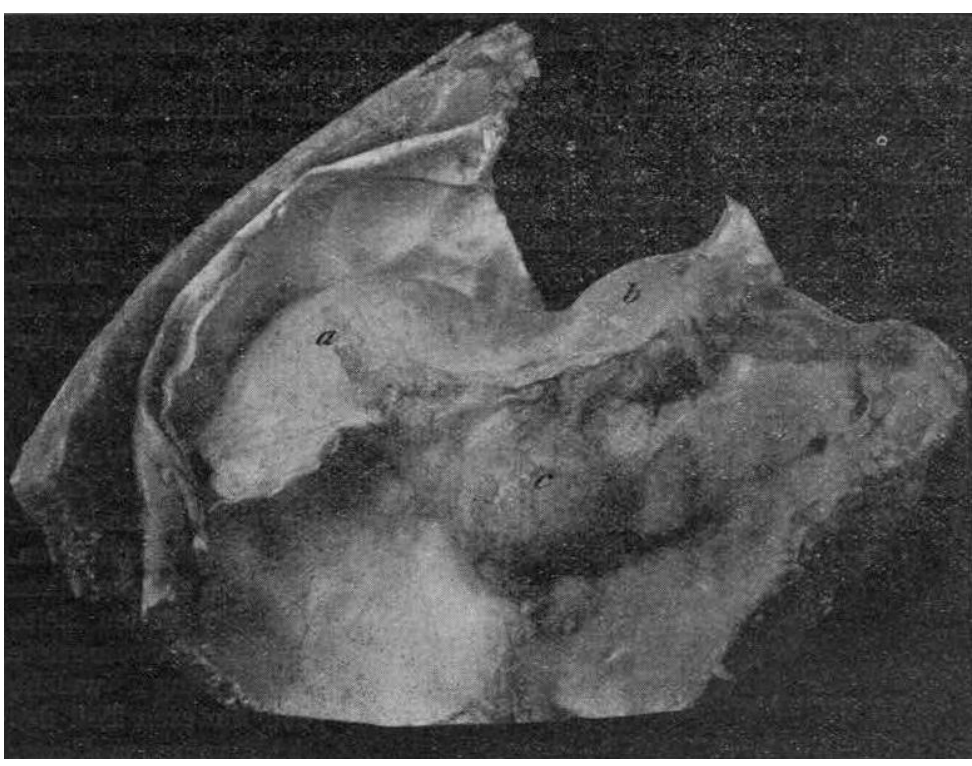

gelegen ist. Dicht an der Spitze des Felsenbeins, entsprechend der oberen Kante desselben fand sich ein dritter, mit einem kurzen breiten Stiel aufsitzender Tumor von der Größe einer Walnuß, der sich gegen die linke Fläche der Brücke vorgedrängt hatte. Dieser Tumor wurde bei der Obduktion behufs mikroskopischer Untersuchung abgetrennt, an dem Präparat ist nur noch die Anheftungsstelle zu erkennen. Ein weiterer kirschengroßer Tumor (c) findet sich an der hinteren Fläche der Pyramide, die Gegend des Porus acusticus internus und dessen Umgebung einnehmend. An der unteren Peripherie des Tumors sieht man ein Bündel von Nervenfasern, dem $\mathrm{N}$. facialis und acusticus angehörend, das offenbar vollständig von der Substanz des Tumors durchsetzt ist. Die verschiedenen Tumoren sind von ziemlich weicher Konsistenz und zum Teil, wie die auch zwischen ihnen gelegenen Partien prall elastisch anzufühlen. Ueber den Tumoren erscheint die Dura mater im wesentlichen unverändert, nur an einzelnen Stellen stark verdünnt. Schließlich ist noch $\mathrm{zu}$ bemerken, daß in der Höhle des am Präparate noch teilweise erhaltenen Keilbeins sich ein fünfter, den betreffenden Teil der Höhle ausfüllender Tumor von ausgesprochen gallertiger Beschaffenheit findet, der, soweit man dies an dem nicht zerlegten Präparate erkennen kann, in den an der Spitze des Felsenbeines gelegenen Tumor übergeht. Die mikroskopische Untersuchung ergab, daß wir es mit einem Myxom resp. Myxo-Fibrom zu tun hatten. Die zum Teil mit Hämatoxylin und Eosin, zum Teil nach va n Gieson gefärbten Schnitte zeigen ein weitmaschiges Bindegewebsnetz, dessen Maschen von den Ausläufern stern- und spindelförmiger Zellen gebildet werden. An einzelnen Stellen sieht man Züge von ausgesprochen fibrillärem Bindegewebe mit reichlich, ebenso wie in den genannten Maschenräumen, wenn auch hier in geringer Menge, eingestreuten polynukleären Leukozyten und neben diesen noch eine ziemlich beträchtliche Anzahl von großen mononukleären Zellen mit gekörntem Protoplasma, die ganz das Aussehen von Myelozyten haben.

Die Frage nach dem Ausgangspunkte der beschriebenen Tumoren dürfte sich mit Sicherheit nicht beantworten lassen. Einmal könnte man daran denken, daß wir es, entsprechend der Lehre Cohnheims von der embryonalen Anlage der Geschwülste, mit einer Neubildung zu tun haben, die ihren Ursprung dem Schleimgewebe verdankt, das bekanntlich im Fötalleben die Paukenhöhle ausfüllt; dagegen spricht jedoch der normale Befund am Trommelfell, denn man hätte doch erwarten müssen, daß der Tumor nicht nur nach innen gegen die Substanz des Felsenbeines, sondern vor allem nach außen gegen die Stelle des geringsten Widerstandes, also gegen Trommelfell und äuBeren Gehörgang hin sich ausgebreitet hätte. Mehr Wahrscheinlichkeit hat die Annahme, namentlich mit Rücksicht auf die bei der mikroskopischen Untersuchung gefundenen Knochenmarksbestandteile (Myelozyten), daß die Tumoren vom Knochenmark der Felsenbeinpyramide ausgegangen waren. BirchHirschfeld hat in seinem Artikel "Myxom" in Eulenburgs Realenzyklopädie darauf aufmerksam gemacht, daß das am Knochen vorkommende Myxom vorzugsweise vom Knochenmark seinen Ausgang nimmt. Ob es möglich sein wird, nach Zerlegung des Präparats, wie ich sie vorzunehmen beabsichtige, Genaueres über diese Frage zu eruieren, muß einstweilen dahingestellt bleiben. (Ich behalte mir vor, hierüber sowie über die noch auszuführende mikroskopische Untersuchung des $\mathbf{N}$. acusticus und des Labyrinths später Näheres mitzuteilen.) Schließlich ist noch zu bemerken, daß ich 
in der Literatur, soweit sie mir zugänglich war, nur zwei Fälle von Myxom am Felsenbein gefunden habe. Ueber den einen Fall findet sich eine kurze Notiz in Rokitanskys Lehrbuch der pathologischen Anatomie (3. Auflage, Bd. 1, S. 167), wo er eine rundliche, an der Dura mater, rings um den rechten Porus acusticus internus haftende, hühnereigroße, sukkulente, gallertige Geschwulst beschreibt, die aus einer höchst zurtfaserigen, von oblongen Kernen durchsetzten Textur in Form eines Maschenwerkes bestand. Einen weiteren Fall erwähnt Politzer in seinem Lehrbuch der Ohrenheilkunde (5. Auflage, S. 642). Er rührt von Verm yne her, der als Ursache einer Erblindung, der nach sieben Jabren vollständige Taubheit folgte, ein Myxo-Fibrom der Schädelbasis beschreibt, welches auf das Labyrinth übergriff.

$$
\text { II. }
$$

Im Anschluß an die Ausfluhrungen des Herrn Schwabach möchte ich einige Worte über die neurologische Seite dieses Falles sagen und Ihnen das Gehirn der Kranken an einem Licht-
Fig. 2.

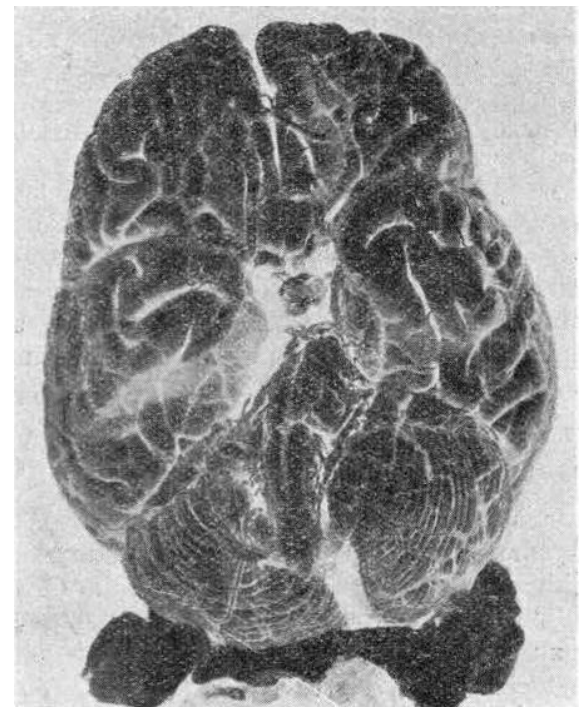
bilde demonstrieren. (cf. Fig. 2.) Sie sehen auf der linken Seite derHirnbasis eine tiefe Einsenkung, welche den hinteren Teil des Schläfenlappens quer durchzieht und bis an die linke Seite des Pons heranreicht. Die linke Seite der Brücke zeigt eine ziemlich tiefe Mulde, und Sie sehen ferner, daß dieser Hirnteil in toto nach rechts hinübergedrängt ist. Auch der proximale Teil der Medulla oblongata ist noch etwas nach rechts hinübergeschoben. SchlieBlich sehen Sie, $\mathrm{da} \beta$ auch die vordere Kante der linken Kleinhirnhemisphäre an der Einsenkung beteiligt ist, und zwar besonders das Gebiet des Flocculus. Legt man das Knochenpräparat in seiner natürlichen Lage auf die Gehirnbasis, so erkennt man, daß die durch die Tumorbildung in toto stark vergrößerte Felsenbeinpyramide genau in die Einsenkung am Schläfenlappen hineinpaßt. Die Mulde am Pons ist auf den Druck der von der Spitze des Felsenbeins ausgehenden knolligen Wucherung zurückzuführen, welche mit dem Knochen selbst nur durch einen kurzen Stiel verbunden war. Durch die anatomischen Verhältnisse werden die klinischen Symptome des Falles vollkommen geklärt.

Wie Sie gehört haben, verlief der Fall ähnlich dem Bilde eines sog. Acusticustumors. Schon zu der Zeit, als die Kranke in die Klinik aufgenommen wurde, war eine multiple Hirnnervenlähmung vorhanden; es bestanden Paresen im ganzen linken Fazialis und linken Abducens; die Hörfähigkeit war auf dieser Seite vollkommen oder fast vollkommen erloschen, während sich im Innervationsgebiete des linken Quintus lebhafte Reizerscheinungen in Form neuralgischer Schmerzen bemerkbar machten. Bei der scharfen Lokalisation der Symptome auf die linke Seite und dem ausgesprochen peripherischen Charakter der Fazialislähmung konnte nur ein basaler Prozeß in der linken hinteren Schädelgrube in Frage kommen; und da die nervöse Schwerhörigkeit von vornherein sehr hervortrat, so ließ sich der Symptomenkomplex zwanglos auf einen primären Acusticustumor zurückführen. Das spätere Hinzukommen der Lähmungserscheinungen im 9., 10., 11. und 12. Hirnnerven konnte man bequem als Druckwirkung einer langsam wachsenden Geschwulst auf die benachbarten Wurzeln deuten. Für die Annahme eines Tumors sprachen dann auch die allgemeinen Hirndrucksymptome, nämlich die Kopfschmerzen und das sich öfter wiederholende Erbrechen. Allerdings waren diese Allgemeinsymptone nicht in der Weise ausgesprochen, wie sie sonst bei Tumoren der hinteren Schädelgrube aufzutreten pflegen; vor allen Dingen fehlte auch die Staunngspapille. Nun sehen Sie, meine Herren, daß unsere Annahme nur zum Teil richtig war; es handelte sich tatsächlich um einen basalen Tumor; aber sein Sitz war nicht im Stamme des Acusticus, sondern im Felsenbein. Die mechanische Wirkung derartiger Felsenbeintumoren auf das Gehirn ist derjenigen der Acusticusgeschwülste ziemlich ähnlich. Hier wie dort haben wir es mit Verdrängungserscheinungen zu tun, und speziell die Dellenbildung an der ventrolateralen Fläche des Pons ist beiden gemeinsam.
Ein besonderes Interesse bietet in dem vorliegenden Falle die Frage, welche mechanischen Faktoren eine so ausgedehnte Hirnnervenlähmung hervorgerufen haben. Die Betrachtung des Präparates gibt darauf eine ziemlich klare Antwort. Es sind offenbar zwei verschiedene Faktoren, welche da in Betracht kommen. Die proximalen der gelähmten Hirnnerven: der Quintus und Abducens, welche nach ihrem Austritt aus dem Pons frontalwärts ziehen, sind durch die Tumormassen an das Zentralorgan angepreßt und dadurch gelähmt worden. Hier handelt es sich also um eine Kompression der Wurzeln an ihren Austrittsstellen aus dem Pons selbst. Für den Fazialis, Acusticus, Glossopharyngeus, Vagus, Accessorius und Hypoglossus kommt aber neben einer leichten Zerrung hauptsächlich eine Kompressionswirkung auf die Nerven bei ihrem Durchtritt durch die Schädelbasis in Betracht. An dem Knochenpräparat erkennen Sie, daß der Porus acusticus internus durch die Geschwulstbildung stark verengt worden ist; und es ist leicht erklärlich, daß die ihn passierenden Nerven an dieser Stelle frühzeitig lädiert werden mußten. Ganz ähnlich liegen die Verhältnisse auch an dem zwischen Felsenbein und Os occipitale gelegenen Foramen jugulare, durch welches der neunte, zehnte und elfte Gehirnnerv den Schädelraum verlassen. Auch bier war bei der Autopsie deutlich zu sehen, daß die Geschwulstmassen den Knochenkanal verengten.

Schwieriger ist die Hypoglossuslähmung zu erklären, welche sich gegen Ende des Krankheitsverlaufes in einer Hemiatrophia linguae mit fibrillärem Zittern bemerkbar machte. Dieser Hirnnerv konnte weder an seiner Austrittsstelle aus der Oblongata noch beim Passieren des Foramen condyloideum in Mitleidenschaft gezogen worden sein. Es ist wahrscheinlich, daß er unmittelbar nach dem Verlassen des Schädels betroffen worden ist, und zwar auf derjenigen Strecke, wo er in der Nachbarschaft des Vagus und der Carotis nach vorn verläuft. Denn man muß annehmen, daß die Tumormassen des Felsenbeins nicht nur nach oben in den Schädelhohlraum hinein gewachsen sind, sondern sich auch etwas nach unten verbreitet haben. Es würde zu weit führen, an dieser Stelle auf die Lähmungssymptome der einzelnen Hirnnerven einzugehen.

Nur auf einen Punkt möchte ich noch hinweisen, weil er von praktischer Bedeutung werden kann, nämlich auf die Differentialdiagnose dieser Schläfenbeingeschwülste von den Tumoren des Kleinhirnbrückenwinkels, insbesondere den Acusticustumoren. Sie wissen, meine Herren, daß die Tumoren der hinteren Schädelgrube, speziell die Ácusticusgeschwülste in den letzten Jahren wiederholt der Gegenstand chirurgischer Eingriffe gewesen sind und da $\beta$ bereits über eine Anzahl günstiger Resultate berichtet worden ist. Myxome, Sarkome oder andere Kıochengeschwülste der Felsenbeinpyramide werden vom Chirurgen kaum in Angriff genommen werden, weil bei ihrer Entfernung eine Läsion der großen Blutsinus und Gefäße unvermeidlich wäre. Eine exakte Differentialdiagnose kann also im gegebenen Falle sehr wünschenswert sein, und es fragt sich, ob beide Geschwulstarten trotz ihrer großen topographischen A ehnlichkeit sich nicht in einzelnen Punkten voneinander unterscheiden. Ich glaube, daß solche Unterschiede bestehen, wenn man sich auch in Anbetracht der Seltenheit der Felsenbeingeschwülste und der deshalb geringen Zahl einschlägiger Beobachtungen noch mit Reserve ausdrücken muß. Es geht aber aus der Literatur und auch aus der Betrachtung des vorliegenden Falles hervor, daß die multiple Hirnnervenlähmung bei den Felsenbeingeschwülsten ein€ viel größere Ausdehnung gewinnt als bei den Tumoren des Kleinhirnbrückenwinkels. In unserem Falle, der allerdings in seiner Art einzig dasteht, waren die letzten acht Hirnnerven der linken Seite betroffen; in einem Falle von Seiffer und Koch (Charitéannalen, Bd. 25), bei dem es sich um ein myelogenes Sarkom des rechten Os petrosum handelte, die letzten sechs. Auch Bruns hebt hervor, daß bei primären oder metastatischen Tumoren in den Knochen der hinteren Schädelgrube sehr ausgedehnte Hirnnervensymptome zustande kommen, während sie bei anders gearteten Tumoren der Basis weniger ausgedehnt sind. Diese Tatsache findet ihre Erklärung darin, daß bei den Knochengeschwülsten die Durchtrittspassagen der Nerven der Schädelbasis frühzeitig verlegt werden. Im Porus acusticus und im Foramen jugulare können sehr leicht zwei resp. drei Hirnnerven zu gleicher Zeit betroffen werden, und in diesen engen Knochenkanälen ist ein Entrinnen gegenüber der andringenden Geschwulstmasse unmöglich. Bei den Tumoren, die sich im Schädelhohlraum entwickeln, können dagegen die Nerven vermöge ihrer eigenen Elastizität und Verschiebbarkeit der andringenden Neubildung ausweichen und trotz scheinbar großer Bedrängnis lange funktionstüchtig bleiben. Ein weiteres Unterscheidungsmerkmal liegt in dem Tempo des Krankheitsverlaufes. Während sich bei Knochengeschwülsten das klinische Bild meist sehr langsam entwickelt, sehen wir bei den Tumoren der Hirnbasis im allgemeinen einen rascheren Verlauf. 
Im engsten Zusammenhang damit steht der dritte Differentialpunkt. Dieser betrifft die allgemeinen Hirndrucksymptome. Bei den Hirngeschwülsten der hinteren Schädelgrube, speziell auch den Acusticustumoren, sind die Allgemeinsymptome im allgemeinen recht erheblich. Insbesondere wird die Stauungspapille nur relativ selten vermißt; bei den Knochengeschwülsten sind dagegen die Hirndruckerscheinungen viel geringer, und die Stauungspapille scheint häufig zu fehlen. Auch dieser Unterschied ist im wesentlichen auf das Tempo des Tumorwachstums zurückzuführen. Bei den langsamer wachsenden Knochenneubildungen kann sich das Gehirn den veränderten Druckverhältnissen leichter anpassen als bei den intrakraniellen Tumoren. Schließlich wäre noch darauf hinzuweisen, daß von den Kleinhirnbrückenwinkeltumoren sehr häufig Nachbarschaftssymptome von seiten des Kleinhirns (cerebellarer Schwindel und Ataxie) ausgelöst werden, welche den Knochentumoren zu fehlen scheinen. - Von der röntgenologischen Untersuchung kann man sich in derartigen Fällen nicht allzuviel versprechen. Die funktionelle Diagnostik leistet da vor der Hand noch mehr, und ihr weiterer Ausbau nach der gekennzeichneten Richtung ist zum mindesten nicht überflüssig. 